University of Nebraska - Lincoln

DigitalCommons@University of Nebraska - Lincoln

Faculty Publications, Department of Physics and Astronomy

Research Papers in Physics and Astronomy

2-13-2006

\title{
Exchange Bias Training Effect in Coupled All Ferromagnetic Bilayer Structures
}

\author{
Christian Binek \\ University of Nebraska-Lincoln, cbinek@unl.edu \\ Srinivas Polisetty \\ Hitachi Global Storage Technologies, polisetty.srinivas@gmail.com \\ Xi He \\ University of Nebraska-Lincoln
}

A. Berger

Hitachi Global Storage Technologies

Follow this and additional works at: https://digitalcommons.unl.edu/physicsfacpub

Part of the Physics Commons

Binek, Christian; Polisetty, Srinivas; He, Xi; and Berger, A., "Exchange Bias Training Effect in Coupled All Ferromagnetic Bilayer Structures" (2006). Faculty Publications, Department of Physics and Astronomy. 20.

https://digitalcommons.unl.edu/physicsfacpub/20

This Article is brought to you for free and open access by the Research Papers in Physics and Astronomy at DigitalCommons@University of Nebraska - Lincoln. It has been accepted for inclusion in Faculty Publications, Department of Physics and Astronomy by an authorized administrator of DigitalCommons@University of Nebraska Lincoln. 


\title{
Exchange Bias Training Effect in Coupled All Ferromagnetic Bilayer Structures
}

\author{
Ch. Binek, ${ }^{1}$ S. Polisetty, ${ }^{2}$ Xi He, ${ }^{1}$ and A. Berger ${ }^{2}$ \\ ${ }^{1}$ Department of Physics and Astronomy and the Center for Materials Research and Analysis, Ferguson Hall, University of Nebraska, \\ Lincoln, Nebraska 68588-0111, USA \\ ${ }^{2}$ San Jose Research Center, Hitachi Global Storage Technologies, San Jose, California, USA
}

(Received 26 October 2005; published 13 February 2006)

\begin{abstract}
Exchange coupled bilayers of soft and hard ferromagnetic thin films show remarkable analogies to conventional antiferromagnetic/ferromagnetic exchange bias heterostructures. Not only do all these ferromagnetic bilayers exhibit a tunable exchange bias effect, they also show a distinct training behavior upon cycling the soft layer through consecutive hysteresis loops. In contrast with conventional exchange bias systems, such all ferromagnetic bilayer structures allow the observation of training induced changes in the bias-setting hardmagnetic layer by means of simple magnetometry. Our experiments show unambiguously that the exchange bias training effect is driven by deviations from equilibrium in the pinning layer. A comparison of our experimental data with predictions from a theory based upon triggered relaxation phenomena shows excellent agreement.
\end{abstract}

PACS numbers: $75.60 .-\mathrm{d}, 75.70 . \mathrm{Cn}$

Exchange bias (EB) is known as a magnetic coupling phenomenon at ferromagnetic (FM)-antiferromagnetic (AFM) interfaces with strong implications for magnetic field sensor applications, read heads, and modern approaches to spintronics [1-6]. It is initialized by field cooling the heterosystem to below the blocking temperature, $T_{B}$, at which AFM order is established, at least on a mesoscopic scale [7]. The most striking and widely studied feature in EB systems is the shift of the FM hysteresis loop along the magnetic field axis by the amount $\mu_{0} H_{\mathrm{EB}}$. Furthermore, a gradual degradation of the EB field can be observed upon cycling the heterostructure through consecutive hysteresis loops [8-12]. This aging phenomenon is know as the training effect (TE) and is quantified by $\mu_{0} H_{\mathrm{EB}}$ vs $n$, where $n$ is the number of loops cycled after first setting the EB via field cooling. EB and its training have been observed in various magnetic systems ranging from core-shell magnetic granules and AFM-FM thin film heterolayers to ferromagnetic nanodomains embedded in an AFM matrix of charge ordered manganites [13-16].

In this Letter, we study the EB training effect in all FM bilayers, in which a magnetically soft CoCr layer (SL) is exchange coupled via a Ru interlayer with a hardmagnetic CoPtCrB pinning layer (HL) (see the inset in Fig. 1). In comparison to the conventionally used AFM layer in EB structures, FM pinning layers provide unique experimental access to the change in their magnetization state and, in turn, reveal the dependence of the bias field on the pinning layer magnetization [17]. Therefore, the cycle dependent evolution of the pinning layer magnetization can be unambiguously measured by means of simple magnetometry and its correlation with training of the bias field is clearly evidenced. Recent attempts to show the analogous correlation between aging of the interface magnetization in an AFM pinning layer and the training of the EB field in AFM-FM heterostructures faced serious difficulties because of the smallness of the excess magnetic moment that gives rise to conventional EB $[15,18,19]$. Also, in these EB systems, proportionality between the moment at the interface and the AFM bulk magnetic moment is a faintly motivated assumption. The latter is far more reasonable in the case of a thin FM pinning layer with a homogeneous spin structure along the normal of the film. We also present a transparent theory of the TE adapted to all FM bilayers, which shows excellent agreement with our experimental data.

Figure 1 (left frame) displays a schematic of our sample structure and its overall magnetic hysteresis $m$ vs $\mu_{0} H$ (dashed line) where $m$ is the magnetic moment and $H$ is the applied magnetic field. The measurements are done at
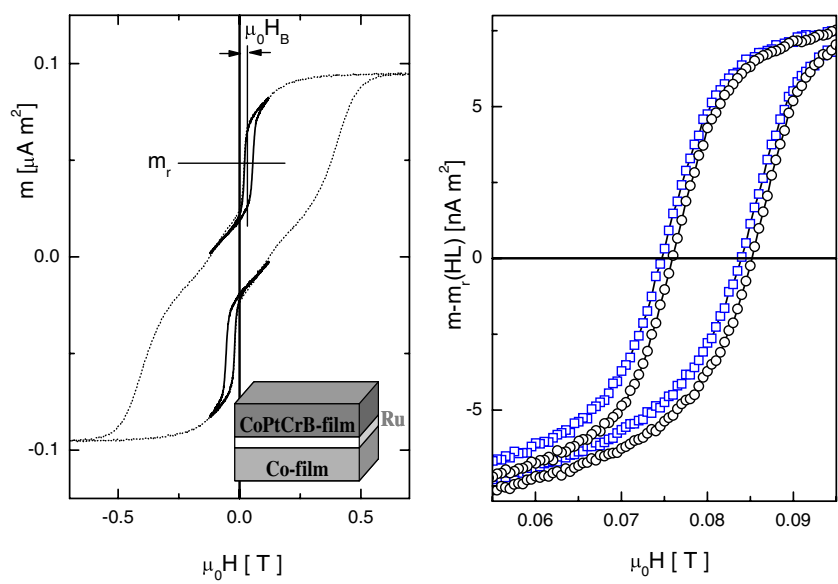

FIG. 1 (color online). Overall magnetic hysteresis $m$ vs $\mu_{0} H$ (left frame, dashed line). Thick solid lines are low field minor loops after positive and negative saturation, respectively. The horizontal line visualizes $m_{r}$ for the upper SL loop, the vertical line indicates the shift of the SL loop along the field axis relative to $H=0$. The inset is a schematic of the sample. The right frame shows the TE between the first (squares) and 20th (circles) hysteresis loop of the $\mathrm{CoCr}$ soft layer after first setting the $\mathrm{CoPtCrB}$ magnetization state. 
room temperature by means of alternating gradient force magnetometry. The shape of the overall loop reflects the well separated switching field distributions of the HL and SL. The thick solid lines are low field minor loops after positive and negative saturation, respectively. The low field response of these minor loops is dominated by the magnetization reversal of the soft magnetic $\mathrm{CoCr}$ layer. Their positions are shifted along the $m$ axis due to the remanent magnetic moment $m_{r}$ of the CoPtCrB layer and along the field axis due to the Ru mediated interlayer exchange field $\mu_{0} H_{B}$. The loop shift along the field axis is denoted as $\mu_{0} H_{B}$ to distinguish it from the EB field, $\mu_{0} H_{\mathrm{EB}}$, in AFMFM systems. Figure 1 (right frame) shows an example of the TE in our all FM bilayers. The measurements were performed after first saturating the bilayer in $\mu_{0} H=0.8 \mathrm{~T}$ and subsequent partial demagnetization of the pinning layer in a static set field of $\mu_{0} H_{\text {set }}=-0.34 \mathrm{~T}$. The minor SL loops are measured in magnetic fields of moderate strength, $0 \leq \mu_{0} H<0.14 \mathrm{~T}$, which do not switch the HL. After subtracting $m_{r}(\mathrm{HL})$, respectively, the first $(n=$ 1 , squares) and 20th $(n=20$, circles) minor hysteresis loops of the SL reveal a clear relative shift along the field axis. Evidently, the bias field experiences training through consecutive magnetization reversals of the SL and increases within the 20 subsequently cycled loops by about $1 \mathrm{mT}$. This TE in $\mu_{0} H_{B}$ is accompanied by the training of the HL magnetization towards an increased equilibrium value. More specific, the shifts of the SL loops along the $m$ and field axis are linearly correlated [17]. In order to analyze the $\mu_{0} H_{B}$ vs $n$ dependence quantitatively we determine the EB field at each $n$ value for a number of different starting conditions.

Figure 2 (left frame) shows the TE of the FM bilayer for 4 different set fields $\mu_{0} H_{\text {set }}= \pm 0.36$ and $\pm 0.34 \mathrm{~T}$ after saturation in $\mu_{0} H=\mp 0.8 \mathrm{~T}$, respectively. When saturating the bilayer in a positive (negative) magnetic field, a negative (positive) set field reduces (increases) the magnetization of the pinning layer. Subsequent SL hysteresis loops will drag the HL back towards equilibrium corresponding to increased (reduced) magnetization. Triangles display the experimental $\mu_{0} H_{B}$ vs $n$ training data. Similar to previous findings for conventional EB systems [15], we observe that the loop-to-loop TE is strongest in the beginning of the measurement procedure, i.e., for low $n$ values, and becomes substantially less pronounced for higher $n$. This also indicates that the related relaxation processes in the biasing HL occur predominantly during the first few reversals, while subsequent SL loops produce only minor changes. In accordance with the symmetry of the overall hysteresis loop (see Fig. 1, left frame) identical absolute values but opposite signs of the saturation and set fields generates symmetry in the corresponding $\mu_{0} H_{B}$ vs $n$ dependence with respect to $\mu_{0} H_{B}=0$. The data in Fig. 2 also show that with increasing absolute value of the set field the resulting absolute bias field decreases, which simply reflects the fact that the absolute value of the HL magnetization is further reduced [20].
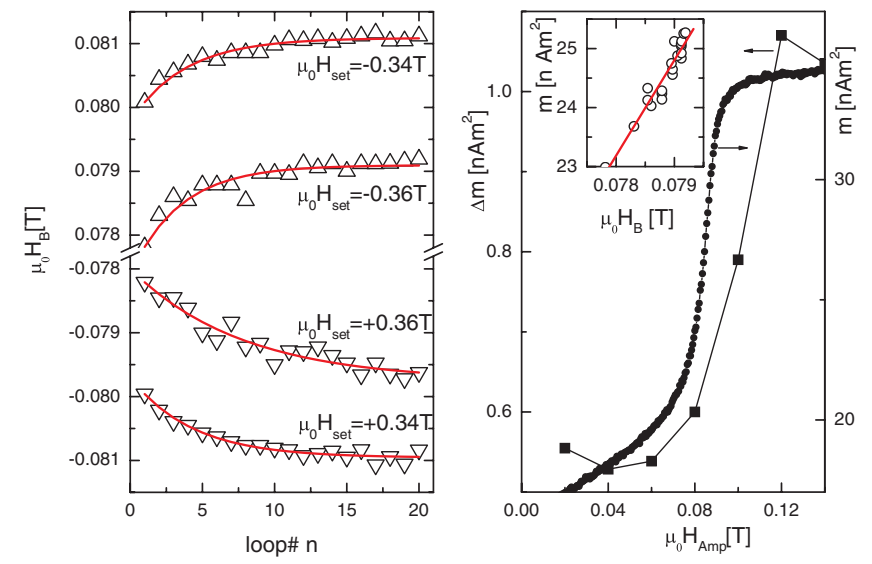

FIG. 2 (color online). Training effect $\mu_{0} H_{B}$ vs $n$ (left frame) of the HL-SL bilayer for set fields $\mu_{0} H_{\text {set }}= \pm 0.36$ and $\pm 0.34 \mathrm{~T}$ after saturation in $\mu_{0} H=0.8 \mathrm{~T}$ for negative and $-0.8 \mathrm{~T}$ for positive set fields, respectively. Experimental data are shown as triangles while lines represent least-squares fits of Eq. (5) to the respective data sets. The right frame shows the change of the TE (squares) for various field amplitudes $\mu_{0} H_{\text {Amp }}$, as measured by the HL remanence magnetization in comparison to the up magnetization branch of the SL hysteresis. The inset (open circles) evidences the linear relation between the remanent magnetization and the bias field in our training experiments. Data are obtained from 20 consecutive loops after saturation at $\mu_{0} H=0.8 \mathrm{~T}$ and initialization in a set field of $\mu_{0} H_{\text {set }}=$ $-0.34 \mathrm{~T}$. The line is the best linear fit to the data.

Given the fact that the time-averaged applied magnetic field in our measurements of the TE is not zero, it is necessary to show experimentally that the TE is indeed triggered by the cycled SL hysteresis loops. In particular, we have to rule out that the change of $\mu_{0} H_{B}$ reflects a continuous relaxation phenomenon depending on the time the HL is exposed to the applied magnetic field. In order to evidence the triggered nature of the TE we study the change of the HL magnetization for 10 subsequent SL loops obtained for various field amplitudes $\mu_{0} H_{\text {Amp }}$. Each set of 10 loops takes place in applied magnetic fields $0 \leq \mu_{0} H \leq \mu_{0} H_{\text {Amp }}$ after the sample has been initialized each time in an identical fashion through saturation of the bilayer in $\mu_{0} H=0.8 \mathrm{~T}$ and partial demagnetization in a set field of $\mu_{0} H_{\text {set }}=-0.34 \mathrm{~T}$. Since the SL does not completely reverse for $\mu_{0} H_{\text {Amp }}$ smaller than the saturation field, a meaningful bias field cannot be determined from the loop itself. However, due to the proportionality between the HL magnetization and $\mu_{0} H_{B}$, we are able to determine the field cycling effect onto the bilayer system by simply measuring the remanent HL magnetization. The proportionality between $m_{r}$ and $H_{B}$, known from Ref. [17] is independently evidenced here. The inset of Fig. 2 (right frame) shows as an example the remanent magnetization $m(H=0)$ of the bilayer for 20 consecutive loops which is proportional to the HL magnetization due to uniform antiparallel alignment of the SL in zero field. Each loop results in an individual value for the remanent magnetization and 
the bias field. Clearly, within the statistical error of the data, the expected linear dependence $m(H=0) \propto \mu_{0} H_{B}$ is confirmed. Therefore the change of the remanent magnetization $\Delta m=m(H=0, n=1)-m(H=0, n=10)$ taking place during a 10 loop training sequence in the field interval $0 \leq \mu_{0} H \leq \mu_{0} H_{\mathrm{Amp}}$ can be used as a measure of the change of the bias field $\Delta H_{B}=H_{B}(n=1)-H_{B}(n=$ 10). Figure 2 (right frame) shows that $\Delta m$ vs $\mu_{0} H_{\mathrm{Amp}}$ (squares) increases significantly at $\mu_{0} H_{\mathrm{Amp}} \approx 0.08 \mathrm{~T}$, simultaneous with the onset of magnetization reversal in the $\mathrm{SL}$. This correlation between hysteretic behavior of the SL and the TE is evidenced when comparing $\Delta m$ vs $\mu_{0} H_{\mathrm{Amp}}$ with the up magnetization branch $m$ vs $\mu_{0} H$ of the hysteresis loop (solid circles). The constant value of $\Delta m$ vs $\mu_{0} H_{\text {Amp }}$ for field amplitudes $0.02 \leq \mu_{0} H_{\text {Amp }}<0.08 \mathrm{~T}$ and $H_{\mathrm{Amp}}>0.12 \mathrm{~T}$ indicates that the TE is not directly caused by the time and amplitude of the applied field, but triggered by the SL magnetization reversal. This result is in accordance with previous findings obtained by polarized neutron scattering, high resolution photoemission electron microscopy, and micromagnetic simulations [21,22].

It is general consensus that training of the EB is caused by the nonequilibrium nature of the spin structure in the pinning layer [12,23-27]. Thus, the gradual decrease of $\mu_{0} H_{\mathrm{EB}}$ with increasing $n$ is a fingerprint of rearrangements in the pinning layer spin structure towards an equilibrium configuration. These general assumptions are corroborated by our experimental observation that virtually no TE is present in our samples if we start the minor loop cycling from a fully magnetized HL state, i.e., beginning very close to the equilibrium configuration of the HL. Significant TE is achieved only when a set field drives the HL out of saturation into a domain state. Consecutively cycled loops of the SL then drive the HL, in part, back towards saturation causing the TE. This qualitative picture is for the first time confirmed by means of magnetometry. It is a conceptual advantage of all FM bilayer systems that the deviation of the HL magnetization from its equilibrium state can be measured via either $m_{r}(\mathrm{HL})$ or the bias field which is proportional to $m_{r}(\mathrm{HL})$. Figure 3 shows the experimentally measured size of the TE, $\left|\mu_{0} H_{B}(n=1)-\mu_{0} H_{B}^{e}\right|$ vs $\left|\mu_{0} H_{B}(n=1)\right|$, i.e., the measurement starting point. Hereby $\mu_{0} H_{B}^{e}$ denotes the equilibrium bias field in the limit of large $n$. According to the proportionality between the HL magnetization and the bias field, the increase of $\left|\mu_{0} H_{B}(n=1)-\mu_{0} H_{B}^{e}\right|$ with decreasing bias field $\mu_{0} H_{B}(n=1)$ evidences that training requires a HL domain state that is not in equilibrium to allow for spin configurational rearrangements towards this very equilibrium state. The more the HL magnetization deviates from its equilibrium state of saturation the more pronounced is the TE.

In the framework of this physical picture, the TE in conventional EB systems has recently been described by means of the Landau-Khalatnikov (LK) theory, which allowed the derivation of a sequence equation that deter-

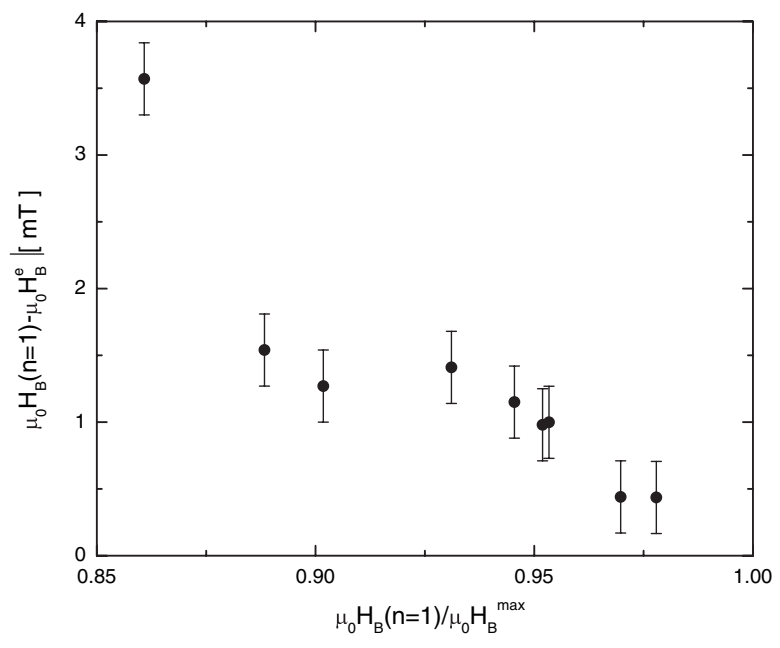

FIG. 3. Dynamics of the TE $\left|\mu_{0} H_{B}(n=1)-\mu_{0} H_{B}^{e}\right|$ measured for various magnetization states of the HL. The HL magnetization at $n=1$ is linearly correlated with the bias field $\mu_{0} H_{B}(n=1)$. The maximum bias field $\mu_{0} H_{B}^{\max } \approx 0.084 \mathrm{~T}$ is achieved when the HL is saturated and no training appears.

mines $\mu_{0} H_{\mathrm{EB}}(n+1)$ from its predecessor [28,29]. Here, we outline the analogous theoretical approach in order to calculate the TE in all FM bilayer systems [30]. Using this LK approach the time evolution of the interface magnetization in the pinning layer $S$ is given by

$$
\xi \dot{S}=-\frac{\partial \Delta F}{\partial S},
$$

where $\xi$ is a phenomenological damping constant, $\dot{S}$ is the time derivative of $S$, and $\Delta F$ the nonequilibrium free energy of the pinning layer. The triggered nature of the TE [compare discussion of Fig. 2 (right frame)] is best taken into account through discretization of the continuous differential equation (1), by replacing $\dot{S}$ with $[S(n+1)-$ $S(n)] / \tau$, with $\tau$ being the relevant experimental time constant. In its simplest form, $\Delta F$ is characterized by a single FM order parameter. A series expansion of $\Delta F$ in the vicinity of the equilibrium value, $S=S_{e}$, has a leading quadratic term

$$
\Delta F \propto(\delta S)^{2},
$$

where $\delta S=S(n)-S_{e}$ quantifies the deviation from the equilibrium interface magnetization during the $n$th loop. Together with Eq. (2) we obtain from the discretized LK equation (1) an implicit sequence equation

$$
S(n+1)=(K+1) S(n)-K S_{e}
$$

where $K$ is a constant containing the proportionality constant involved in (2) as well as $\xi$ and $\tau$, that can be used as fitting parameter. Hereby, numerical values for $K$ need to be limited to $-1<K<0$ in order to facilitate convergence. A further inspection of Eq. (3) actually allows us to derive an explicit expression for $S(n)$ 


$$
S_{n}=(K+1)^{n-1} S_{1}-K S_{e}(K+1)^{n} \sum_{j=2}^{n}\left(\frac{1}{K+1}\right)^{j} .
$$

Taking into account that $S \propto m_{r}$ for our thin FM biasing films $[17,20]$ and the already demonstrated proportionality $m_{r} \propto \mu_{0} H_{B}$ one obtains an explicit expression for the cycle dependent bias field

$$
\begin{aligned}
\mu_{0} H_{B}(n)= & (K+1)^{n-1}\left\{\mu_{0} H_{B}(1)\right. \\
& \left.-K \mu_{0} H_{B}^{e}\left[\frac{(K+1)^{n+1}-1}{K(K+1)^{n-1}}-(K+2)\right]\right\} .
\end{aligned}
$$

Figure 2 (left frame, lines) shows the results of leastsquares fits of Eq. (5) to our experimental data sets. Evidently, there is excellent agreement between the here derived theoretical expression and our experiments, validating our theoretical approach. In our analysis, $K$ and the equilibrium bias field $\mu_{0} H_{B}^{e}=\mu_{0} H_{B}(n \rightarrow \infty)$ enter Eq. (5) as fitting parameters while $\mu_{0} H_{B}(n=1)$ is fixed as being the bias field of the first loop. Note that the line has physical meaning only at integer values. Furthermore, it is remarkable that the parameter $K$ varies only by about $25 \%$ around $K=-0.2$ throughout all fits indicating that $K$ is virtually independent from the field initialization. In contrast to the here discussed FM-FM systems, the analysis of AFM-FM systems gives rise to a free energy expression with a leading term of 4th order in $\delta S=S(n)-S_{e}$, resulting in a slower power law approach to equilibrium, a fact that was previously confirmed experimentally [28,29].

In summary, we have shown that FM bilayers posses prototypical properties for the fundamental understanding of exchange bias and demonstrated for the first time its corresponding training phenomenon. From an experimental standpoint, these systems are vastly superior to conventional exchange bias systems due to the FM nature of the pinning layer. The latter allows a characterization of its magnetization state by means of simple magnetometry, enabling us to unambiguously demonstrate that the deviations from equilibrium in the pinning layer are the driving force behind the exchange bias training effect. We furthermore derive a theoretical description of the training effect for such all ferromagnetic bilayer systems based upon the discretized dynamical LK equation. The resulting equation shows excellent quantitative agreement with our experimental data, corroborating the underlying physical picture of the training effect as a triggered relaxation mechanism towards the equilibrium state of the pinning layer.

We thank NSF-MRSEC for financial support.

[1] G. Prinz and K. Hathaway, Phys. Today 48 No. 4, 24 (1995).
[2] Ch. Binek and B. Doudin, J. Phys. Condens. Matter 17, L39 (2005).

[3] J. Nogués and I. K. Schuller, J. Magn. Magn. Mater. 192, 203 (1999).

[4] A. Berkowitz and K. Takano, J. Magn. Magn. Mater. 200, 552 (1999).

[5] R. Stamps, J. Phys. D 33, R247 (2000).

[6] M. Kiwi, J. Magn. Magn. Mater. 234, 584 (2001).

[7] I. Roshchin, O. Petracic, R. Morales, Li Zhi-Pan, X. Batlle, and I. K. Schuller, Europhys. Lett. 71, 297 (2005).

[8] D. Paccard, C. Schlenker, O. Massenet, R. Montmory, and A. Yelon, Phys. Status Solidi 16, 301 (1966).

[9] C. Schlenker, S. S. P. Parkin, J. C. Scott, and K. Howard, J. Magn. Magn. Mater. 54, 801 (1986).

[10] K. Zhang, T. Zhao, and M. Fujiwara, J. Appl. Phys. 89, 6910 (2001).

[11] S. G. te Velthuis, A. Berger, G.P. Felcher, B. Hill, and E. Dahlberg, J. Appl. Phys. 87, 5046 (2000).

[12] A. Hoffmann, Phys. Rev. Lett. 93, 097203 (2004).

[13] W.H. Meiklejohn and C.P. Bean, Phys. Rev. 105, 904 (1957).

[14] V. Skumryev, S. Stoyanov, Y. Zhang, G. Hadjipanayis, D. Givord, and J. Nogués, Nature (London) 423, 850 (2003).

[15] A. Hochstrat, Ch. Binek, and W. Kleemann, Phys. Rev. B 66, 092409 (2002).

[16] D. Niebieskikwiat and M. B. Salamon, Phys. Rev. B 72, 174422 (2005).

[17] A. Berger, D. T. Margulies, and H. Do, Appl. Phys. Lett. 85, 1571 (2004).

[18] H. Ohldag, A. Scholl, F. Nolting, E. Arenholz, S. Maat, A. T. Young, M. Carey, and J. Stöhr, Phys. Rev. Lett. 91, 017203 (2003).

[19] P. Kappenberger, S. Martin, Y. Pellmont, H. J. Hug, J. B. Kortright, O. Hellwig, and Eric E. Fullerton, Phys. Rev. Lett. 91, 267202 (2003).

[20] A. Berger, Ch. Binek, D. T. Margulies, A. Moser, and E. E. Fullerton, Physica (Amsterdam) 372B, 168 (2006).

[21] M. Toney, J. Borchers, K. O'Donovan, C. Majkrzak, D. Margulies, and Eric E. Fullerton, Appl. Phys. Lett. 86, 162506 (2005).

[22] L. Thomas, J. Luning, A. Scholl, F. Nolting, S. Anders, J. Stohr, and S. Parkin, Phys. Rev. Lett. 84, 3462 (2000).

[23] U. Nowak, K.D. Usadel, J. Keller, P. Miltényi, B. Beschoten, and G. Güntherodt, Phys. Rev. B 66, 014430 (2002).

[24] D. Suess, M. Kirschner, T. Schrefl, J. Fidler, R. L. Stamps, and J.-V. Kim, Phys. Rev. B 67, 054419 (2003).

[25] M. D. Stiles and R. D. McMichael, Phys. Rev. B 60, 12950 (1999).

[26] H. Xi and R. M. White, Phys. Rev. B 64, 184416 (2001).

[27] S. Brems, D. Buntinx, K. Temst, C. Van Haesendonck, F. Radu, and H. Zabel, Phys. Rev. Lett. 95, 157202 (2005).

[28] Ch. Binek, Phys. Rev. B 70, 014421 (2004).

[29] Ch. Binek, Xi He, and S. Polisetty, Phys. Rev. B 72, 054408 (2005).

[30] G. Vizdrik, S. Ducharme, V. M. Fridkin, and G. Yudin, Phys. Rev. B 68, 094113 (2003). 